\title{
The importance of visual angle in word recognition: A "shrinking screen" modification for visual displays
}

\author{
TIMOTHY JORDAN and CHRISTOPHER MARTIN \\ University of Reading, Whiteknights, Reading, England
}

\begin{abstract}
The visual angle subtended by alphabetic stimuli seems to be given little regard in a large proportion of reading research. Existing empirical evidence suggests that this disregard may be unwise. We describe a modification to video monitors and oscilloscopes that allows the screen size of stimuli to be varied and permits words to be displayed at appropriate visual angles with accuracy and comparative ease. Other advantages of these modifications are also discussed.
\end{abstract}

Empirical examination of the word recognition process has been continuing for 100 years, yet the precise nature of this fundamental human ability is still unknown. One factor that may impede the acquisition of a coherent body of knowledge in this area of psychology is the apparently arbitrary decision, taken by many researchers, concerning the visual angle subtended by stimuli.

When presenting alphabetic stimuli, even slight variations in the horizontal angle occupied by adjacent letters appears to influence the way in which these stimuli are processed. Using multiletter arrays, C. W. Eriksen and his co-workers showed that the influence of adjacent letters upon the perception of a target letter is dramatically reduced when the angular separation of these letters is increased to $1^{\circ}$ or more (B. A. Eriksen \& C. W. Eriksen, 1974; C. W. Eriksen \& Hoffman, 1972, 1973). Furthermore, at separations over $1^{\circ}$, no additional effect is observed. For example, using letters with a horizontal visual angle of approximately $0.25^{\circ}, B$. A. Eriksen and C. W. Eriksen (1974) observed that the time taken to identify a target letter decreased when interletter distance was increased from $0.06^{\circ}$ to $0.50^{\circ}$, and when this increase was from $0.50^{\circ}$ to $1.0^{\circ}$. In addition, C. W. Eriksen and Hoffman (1972), using letters of $0.20^{\circ}$ in width, similarly found that the time taken to identify a target letter decreased when interletter distance was increased from $0.53^{\circ}$ to $1.0^{\circ}$. However, these authors also found that increases in separation from $1.0^{\circ}$ to $1.4^{\circ}$ had no effect on performance.

These results are not obtained with all types of stimuli and therefore do not appear to be due to reductions in sim-

This work was supported by Grant G8408210N from the British Medical Research Council, and by the facilities of the Technical Department of the University of Reading Psychology Department. Many thanks to Bernard Moulden and Philip T. Smith for helpful discussions, to Peter McClatchie for performing the oscilloscope modification, and to four anonymous reviewers for their comments on an earlier version of this paper.

Requests for reprints should be sent to Timothy Jordan, Department of Psychology, University of Reading, Whiteknights, Reading RG6 2AL, England. ple contour interference. Typically, the effect of simple contour interference is found only where interfering contours are separated by less than one third of a degree of visual angle (C. W. Eriksen \& Rohrbaugh, 1970; Flom, Weymouth, \& Kahneman, 1963). Yet in two studies using letter displays (B. A. Eriksen \& C. W. Eriksen, 1974; C. W. Eriksen \& Hoffman, 1972), noise letters proved effective at interletter separations of at least half a degree. Furthermore, C. W. Eriksen and Hoffman (1972) found that, when separated from a target letter by less than $1^{\circ}$, black discs produce far less interference than do their noise-letter counterparts, yet C. W. Eriksen and Rohrbaugh (1970) found that such discs show similar interference effects to letters when they are presented sufficiently close to a target leter.

Although the importance of controlling the angular separation between letters is implicated by the evidence presented thus far, the interference found only when adjacent letters occupy horizontal visual angles of up to $1^{\circ}$ also suggests that a "spotlight" of visual attention exists for all letters presented within $1^{\circ}$ of visual angle. More important, this implies that when the amount of orthographic information within this spotlight differs from that found in natural reading situations, experiments using such unnatural stimuli may not be interrogating the natural process of reading. Evidence from tachistoscopic displays of words and nonwords supports this pessimistic view.

The reported fact that briefly presented words are superior to nonwords is an established phenomenon (Cattell, 1886), which more recent research suggests is due to the relative perceptibility of these two types of stimulus (Johnston, 1978; Reicher, 1969; Wheeler, 1970). In examining the part played by visual angle in this effect, Purcell, Stanovich, and Spector (1978) reported that in backward-masked tachistoscopic displays, when threeletter stimuli subtend a visual angle of less than $0.60^{\circ}$, words are reported more accurately than are matched nonwords. However, this effect is removed when these same word and nonword items are presented at a visual angle of over $2^{\circ}$. Furthermore, when reviewing the relevant 
literature, Purcell et al. (1978) found that the word superiority effect tended to be correlated with visual angle, occurring predominantly when stimuli subtended relatively small amounts of visual angle. This led the authors to conclude that the processing of words may be fundamentally different at different visual angles. More specifically, features that are used to read words occupying smaller visual angles (approximately 4 characters/deg) may be disrupted when these same stimuli subtend visual angles that are comparatively large.

The perception of words at these smaller visual angles appears to be part of natural reading. In everyday reading behavior, alphabetic material is read at distances that bring approximately four characters within $1^{\circ}$ of visual angle. This informal evidence is in agreement with conclusions drawn from eye movements research, which also suggest that three or four characters are brought within $1^{\circ}$ of visual angle when reading (Rayner \& Bertera, 1979).

It seems that evidence suggesting that aphabetic stimuli are processed in a qualitatively different way at smaller visual angles, where four characters occupy $1^{\circ}$ of visual angle, is in agreement with natural reading behavior, and may reflect processes that are employed in natural reading, but that may be prevented in experiments where stimuli subtend excessive visual angles. Despite this evidence and its implications for research, many studies of visual word recognition make no mention of the visual angles subtended by their stimuli. In recent studies where this information is given, the way in which we recognize words has been examined using four-letter stimuli occupying $1.50^{\circ}$ (Massaro \& Klitzke, 1979), $2.25^{\circ}$ (Adams, 1979), $2.50^{\circ}$ (Besner \& Swan, 1982), 3.00 (Rumelhart \& McClelland, 1982), and 3.90 (Solman, May, \& Schwartz, 1981) of visual angle. From the arguments and evidence presented thus far, the formation of a cohesive theory of reading based on the use of stimuli occupying this range of visual angles is not only difficult but the resulting theory may also be misleading.

Unfortunately, attempting to control visual angle by changes in viewing distance is not a satisfactory technique. As described by Leibowitz (1971), the initiation of oculomotor movements for focusing stimuli is coupled with a subjective expectation that this action will be accompanied by a change in the size of the retinal image. The influence that this expectation has on the perception of size was demonstrated by Leibowitz and Moore (1966), who showed that different viewing distances alter the perceived extent of a stimulus, even though the stimulus in their experiment always occupied the same visual angle $\left(1^{\circ}\right)$. Leibowitz and Moore (1966) and Leibowitz, Shiina, and Hennessy (1972) also found that oculomotor adjustments influenced the perception of size only at viewing distances of $1 \mathrm{~m}$ or less; at greater viewing distances, the perceived visual angle of a stimulus follows the size of the retinal image. The implications of these findings for the perception of words are presently unknown; for precisely that reason, however, they should not be ignored. Because of natural reading distances and the loss of oculomotor in- fluence at distances of greater than $1 \mathrm{~m}$, maintaining viewing distances at under $1 \mathrm{~m}$ is preferable to variations about the $1-\mathrm{m}$ boundary. However, this restriction not only severely limits the manipulation of visual angle but fails to remove the potentially troublesome influence of different viewing distances and permits the confounding variations in resolution which are often associated with changes in viewing distance, especially for pixelated computergenerated displays.

When word recognition research is conducted to examine the way in which words are naturally read, reasonable precautions should be taken to ensure that the characteristics of a display are well matched to the characteristics that these same stimuli would possess in natural reading situations. Control over visual angle should be one of these precautions, and a simple way of altering the physical size of stimuli would make this control more accessible and, hopefully, more widely used.

Two simple modifications for visual displays are described which permit the precise and simple control of stimulus size in a wide range of laboratory situations. Because the majority of current word recognition research employs computer-generated graphics, these modifications are provided specifically for video monitor and oscilloscope displays.

\section{VIDEO MONITOR MODIFICATION}

\section{Screen Width}

Monochrome video monitors have a width control coil that determines the effective width of the screen. By increasing the number of windings on this coil, screen width can be reduced to a range that is appropriate for a particular laboratory situation. The precise width of the screen within this range is determined by the position of the iron dust core that passes through the center of the coil. By placing a suitable switch in parallel with the added windings of the coil, normal screen width can easily be returned.

\section{Screen Height}

For height adjustment, a variable potentiometer is wired in series with the internal preset height control of the monitor. This method allows variable height adjustment; however, if a fixed change in height is required, the variable potentiometer can be replaced by a preset, which is wired in parallel with a switch. For convenience, the height adjuster can be located on the outside of the monitor cabinet.

\section{Adjusting Screen Size}

To set up the reduced screen size, the width adjuster switch should be set to the "shrunken screen" position and connected to a video signal providing a suitable test stimulus (e.g., an alphabetic string or a crosshatch test pattern). Using a plastic hexagonal trimming tool, the core of the width adjuster is turned counterclockwise (outwards) to reduce screen width to the desired size. To adjust vertical height, the preset is set to the mid position. 
Height adjustment is then achieved using the height adjuster fixed to the outside of the monitor cabinet.

On the modified monitors, the reduced screen is as central as the original. However, the vertical and horizontal hold controls can be used to center exactly the reduced screen.

Using this modification on a Hitachi VM-900E, a 2-m winding of single core insulated wire $(0.6 \mathrm{~mm}$ diam) reduced a diagonal screen size of $230 \mathrm{~mm}$ to $152 \mathrm{~mm}$. Greater reduction can be achieved by inserting a second iron dust core into the width control coil. With two cores, a diagonal screen size of $56 \mathrm{~mm}$ is achieved, but even this can be improved upon by using more windings on the width control coil and an appropriately adjusted height control. Even at the smallest screen size, the clarity of the image is exceptionally good.

\section{OSCILLOSCOPE MODIFICATION}

Oscilloscopes incorporate a limited form of screen size adjustment in the gain controls for the $x$ and $y$ axes of the display. Therefore, the shrinking screen modification for oscilloscopes is very much simpler than for video monitors, and involves altering the range over which the external gain controls will operate. This is achieved by inserting presets in the oscilloscope's own $x$ and $y$ amplifier gain control circuits. In this way, a suitable range of screen sizes can be obtained.

The desired character size of the display is obtained by setting the value of each of the two presets until the appropriate width and height of the display are achieved. Because of the external $x$ and $y$ gain controls that are fitted as standard, the precise setting of the internal presets is not crucial, because fine alterations to display size can be achieved once the internal presets have brought screen size to within an appropriate range.

As with the monitor modification, eccentricity is not a problem: the $x$ and $y$ position controls centralize the reduced screen.

Using $4 K 7 \Omega$ presets, characters of $16 \mathrm{~mm}$ diam on an unmodified Hewlett-Packard 1332A display were reduced to a diameter of $2 \mathrm{~mm}$ after the shrinking screen modification had been made. This provided an exceptionally clear image. Greater screen reduction is possible by using presets of a larger resistance.

\section{DISCUSSION}

With these modifications, video monitors and oscilloscopes can be positioned at normal reading distances and used to present alphabetic stimuli occupying precisely controlled visual angles. Because the adjustment of visual angle can be made with minimal effort, by simple adjustments to the size of the display, a potential obstacle to an understanding of the process of word recognition can be removed.

The major role of this modification is to enable alphabetic stimuli to be presented at normal visual angles in a wide range of experiments, allowing a more homogeneous, and readily contrastible, pool of information to be formed in this area of research. However, where visual angle itself is a variable, this factor can be manipulated independently of the perceived resolution of stimuli. When using pixelated displays, shrinking the screen to a sufficient degree enables adjacent pixels in a character to be brought sufficiently close together so that increasing the distance between the centers of these pixels increases the size of the stimulus without affecting the apparent resolution of the display. When using line-drawn computer displays, apparent resolution should not vary so much, but, if required, the resolution of a line-drawn display can be manipulated independently of visual angle in a way similar to that used for pixelated graphics. This facility permits one character set to provide displays over a range of different visual angles and yet still remain matched for viewing distance and the appearance of its contours.

Another generally applicable benefit of the shrinking screen modification is that screen resolution can be dramatically increased without the need to replace the basic components of a laboratory system. Thus, if the size of a stimulus is reduced in order to present it at a natural reading distance and at a natural visual angle, a gain in resolution may also be obtained if the coarseness of the original character set is undesirable. (If visual angle is a within-experiment manipulation, then the resolution of both visual displays should be matched as described above.)

Finally, the shape of a character is not restricted by the resolution of the $x$ and $y$ axes of the computer-generated display. By using the vertical and horizontal screen-size controls, the vertical and horizontal dimensions of a character set can be altered. For example, increasing the vertical size of the screen heightens letters and leaves their widths unchanged. Yet the physical extent of these changes in shape is not restricted to units of one pixel, because changes to the size of the screen directly affect the displacement of the electron beam of the CRT.

\section{REFERENCES}

AdaMs, M. 1. (1979). Models of word recognition. Cognitive Psychol ogy, 11, 133-176

Besner, D., SWAN, M. (1982). Models of lexical access in visual word recognition. Quarterly Journal of Experimental Psychology, 34A, 313-325.

Cattell, J. M. (1886). The time taken up by cerebral operations. Mind, 11, 220-242.

ERIKSEN, B. A., \& ERIKSEN, C. W. (1974). Effects of noise letters upon the identification of a target letter in a nonsearch task. Perception \& Psychophysics, 16, 143-149.

Eriksen, C. W. \& Hoffman, J. E. (1972). Temporal and spatial characteristics of selective encoding from visual displays. Perception \& Psychophysics, 12, 201-204.

ERIKSEN, C. W. HOFFMAN, J. E. (1973). The extent of processing of noise clements during selective encoding from visual displays. Perception \& Psychophysics, 14, 155-160.

Eriksen, C. W. Romrbaugh, J. W. (1970). Some factors determining efficiency of selective attention. The American Joumal of $P_{s y}$ chology, 83, 330-342. 
Flom, M. D., Weymouth, F. W., \& Kahneman, D. (1963). Visual resolution and contour interaction. Journal of the Optical Society of America, 53, 1026-1032.

Johnston, J. C. (1978). A test of the sophisticated guessing theory of word perception. Cognitive Psychology, 10, 123-153.

Leibowitz, H. W. (1971). Sensory, learned and cognitive mechanisms of size perception. Annals of the New York Academy of Sciences, 188 , 47-62.

Leibowitz, H. W., \& MoOre, D. (1966). Role of changes in accommodation and convergence in the perception of size. Journal of the Optical Society of America, 8, 1120-1123.

Leibowitz, H. W., Shinna, K., \& Hennessy, R. T. (1972). Oculomotor adjustments and size constancy. Perception \& Psychophysics, 12, 497-500.

MAsSARo, D. W., \& KLITzKe, D. (1979). The role of lateral masking and orthographic structure in letter and word recognition. Acta Psychologica, 43, 413-426.

Purcell, D. G., Stanovich, K. E., \& Spector, A. (1978). Visual angle and the word superiority effect. Memory \& Cognition, 6, 3-8.
Rayner, K., \& Bertera, J. H. (1979). Reading without a fovea. Science, 206, 468-469.

Reicher, G. M. (1969). Perceptual recognition as a function of meaningfulness of stimulus material. Journal of Experimental Psychology, 81, 275-280.

RumelharT, D. E., \& McClelland, J. L. (1982). An interactive activation model of context effects in letter perception: Part 2 . The contextual enhancement effect and some tests and extensions of the model. Psychological Review, 89, 60-94.

Solman, R. T., MaY, J. G., \& Schwartz, B. D (1981). The word superiority effect: A study using parts of letters. Journal of Experimental Psychology: Human Perception \& Performance, 7, 552-559.

Wheeler, D. (1970). Processes in word recognition. Cognitive Psychology, 1, 59-85.

(Manuscript received October 7, 1986; revision accepted for publication January 21, 1987.) 